\title{
Introduction to ACM Transactions on Social Computing
}

The most generally impactful development in computing in the last decade has been social computing, that is, systems in which users interact, directly or indirectly, with what they believe to be other users or other users' contributions. The most prominent examples of this phenomenon are social media systems-such as Twitter, FaceBook, Pinterest, WeChat, and a host of others. But social computing encompasses many other systems of significant societal import: user-generated content systems, such as Wikipedia or YouTube; crowd sourcing and citizen science systems, such as Amazon Mechanical Turk and the Zooniverse; recommender systems, found on nearly all ecommerce sites; and many others. Despite the diversity of modern social systems, they share many common features and collectively have revolutionized the ways we share and gather information, interact, do business, play games, and support others. These developments attract great interest from researchers. And, importantly, many systems were the product of earlier research that developed and explored computing support for new modes of social interaction.

We are excited to present this inaugural issue of ACM Transactions on Social Computing (TSC, http://tsc.acm.org/), a journal created as a venue for the burgeoning field of social computing. The journal welcomes studies employing a wide range of methods to advance the tools, techniques, understanding, and practice of social computing. It seeks to publish work that covers the full spectrum of social computing, including theoretical, empirical, policy, design, systems, and methodological research contributions. To illustrate, we provide a few examples of possible topics:

- Theoretical

- Foundational algorithmic analyses that account for human and machine data and runtime complexity

- The influence of scale; how differing scales of human and machine participation changes the designs and adoptions of systems

- Empirical

- Motivations for contributing to and participating in social computing systems, both intrinsic and extrinsic

- Communications patterns in online communication forums

- Learning in social computing settings

- Ethnographic studies of social computing in situ

- Policy

- Social impacts of social computing use, such as Internet addiction or spread of misinformation, leading to recommendations to mitigate harms and enhance benefits.

- Ethical and policy issues in social computing

- Design

- Tools that help users understand the individual and collective roles of participants in social computing systems

- Algorithms for personalization within a social computing context, including recommender systems and social matchmaking systems

- Security and privacy mechanisms-both formal and interactive-related to social computing data and systems

2018 Copyright is held by the owner/author(s).

2469-7818/2018/02-ART1e

https://doi.org/10.1145/3181713 
- Social aspects of block chain technologies

- The roles of artificial agents (aka bots) in social computing spaces, including the design, creation, and management of those agents relative to social interactions within a social computing system

- Systems

- System architectures and infrastructure for developing social computing platforms

- Microtasking systems based on techniques for decomposing complex activities into recomposable tasks that can be completed by mixtures of people and machines

- Crowd sourcing, collaborative content creation, productive social gaming, or citizen science systems that include mechanisms to aggregate individual contributions for a collective goal

- Methodological

- Algorithms and approaches for extracting knowledge from social computing usage data and artifacts

TSC has been several years in the making, and it is gratifying to see it now come to life. It is the product of much hard work and we thank the research community for its support. Special thanks go to David McDonald, the founding editor-in-chief, and to the inaugural Board of Associate Editors, who believed in the journal and have given their time and effort to steward high-quality articles through to publication. Their names and affiliations appear on the journal masthead page. We also thank the ACM Publications Board (co-chair Joseph A. Konstan in particular) and headquarters staff for their continuing and invaluable support.

Finally, thank you, readers, for reading this first issue. The articles included are the first four that were accepted. They illustrate the breadth of research that make up the field of social computing. Future issues will include a similar mix, with special issues planned (and proposals welcomed). We hope that you like the variety, novelty, and quality of what you see and that you yourself will want to contribute. We are open to your feedback as we continue to grow and improve the journal over time.

Kevin Crowston

Editor-in-Chief 